\title{
Multi source asynchronous information fusion technology based on cross prompts and its application
}

\author{
Wei Daozhi, ${ }^{*}$, Huang Da, Huang Shucai \\ Air Force Engineering University, 710051 shanxi, xian
}

\begin{abstract}
Several modern local high-tech wars have shown that the development of war is determined by the precise strike weapons at both ends of attack and defense. In this paper, the urgent requirement of defense ballistic missile target is taken as traction, and the heterogeneous multi-sensor cross-prompt technology is used as support to study the heterogeneous multi-sensor cross-prompt and its application in target detection. On the basis of fully studying the basic theory of heterogeneous multi-sensor cross-prompting, a cross-prompting network structure model based on typical anti-missile combat mission driving and a data fusion model based on asynchronous information are established, and a multi-sensor cross-prompting method based on fuzzy decision is designed. The model and method proposed in this paper are applied to target detection and tracking, and the model and method involved in this paper are verified by simulation.
\end{abstract}

\section{Introduction}

After generalization in nonlinear systems, the basic Kalman filtering becomes Unscented Kalman filtering. In this algorithm, a posterior probability density can be obtained by deterministic sampling. The density can be used as an approximate value of a nonlinear function. In the process of calculating statistics, it should satisfy the accuracy requirement of order greater than or equal to 2 . However, there is still a drawback, that is, the higher the state dimension is, the greater the amount of computation is, and sometimes increases more obvious, seriously affecting the real-time performance of the algorithm. In addition, when the non-linear and nonGaussian characteristics are strong, if the filter processes first-order and second-order statistical information, the filtering performance of the filter will rapidly decline, and even more, it will show a divergent trend. Compared with the basic Kalman filtering algorithm, the adaptive filtering algorithm has obvious advantages. It can adjust the prediction residual, which contains the correction of the unmeasured information, so it can effectively deal with the impact of abnormal prediction information on state estimation, and can minimize the error. In the algorithm, robust filtering estimates the robust of the actual observation data, which contains observation noise and interference signals. By calculating, the estimation of useful parameters can be obtained, which can minimize the error in the case of observation anomalies and obtain better state estimation. In this chapter, on the basis of the heterogeneous multi-sensor network model, data fusion model and multi-agent negotiation cross-prompt method, the robust adaptive Unscented Kalman filter is applied to solve the target detection problem of multi-sensor cross-prompt system, and the optimal algorithm about the target is obtained. Accurate information can achieve satisfactory results [1].

\section{2 adaptive filtering}

\section{1 construction of adaptive factors}

The adaptive factor model is divided into the zeroing function model and the non return zero function model $[2,3]$. According to the definition of the return-to-zero function, a statistic is defined in the return-to-zero function model, when the statistic is greater than a given threshold, the adaptive factor is returned to zero; in the non-return-to-zero function model, the adaptive factor is inversely correlated with the discriminate statistic, and when the statistic is larger, the adaptive factor becomes smaller, eventually one by one. Gradually approaching zero.

Suppose the nonlinear system is:

$$
\begin{gathered}
\boldsymbol{x}_{k}=f\left(\boldsymbol{x}_{k-1}\right)+\boldsymbol{w}_{k-1} \\
\boldsymbol{z}_{k-1}=h\left(\boldsymbol{x}_{k-1}\right)+\boldsymbol{v}_{k-1}
\end{gathered}
$$

In the formula, $\mathrm{K}$ is the time, $\boldsymbol{x}_{k}$ is the $\mathrm{n}$ dimensional state vector, $\boldsymbol{z}_{k-1}$ is the m-dimensional measurement vector, $f()$ and $h()$ are the nonlinear state transition function and measurement function, $w_{k}$ is the n-dimensional zero-mean process noise vector, $v_{k}$ is the m-dimensional zero-mean measurement noise.

Where

$$
\overline{\boldsymbol{x}}_{k}=f\left(\boldsymbol{x}_{k-1}\right)
$$

\footnotetext{
a Corresponding author: author@e-mail.org
} 
is $\mathrm{k}$ time status prediction information.

The predicted residual state contains the state corrected by the unmeasured information, which can better reflect the disturbance of the dynamic system. Therefore, the error discriminant statistics and adaptive factors of the state model are constructed with predictive residual as variables $[4,5]$.

The predicted residual $\bar{V}_{k}$ is expressed as

$$
\overline{\boldsymbol{V}}_{k}=h\left(\overline{\boldsymbol{x}}_{k}\right)-\boldsymbol{z}_{k}
$$

Constructing a state model error discriminant statistic using predictive residual $\overline{\boldsymbol{V}}_{k}$ [152]:

$$
\Delta \overline{\boldsymbol{V}}_{k}=\sqrt{\left(\left(\overline{\boldsymbol{V}}_{k}\right)^{\mathrm{T}} \overline{\boldsymbol{V}}_{k} / \operatorname{tr}\left(\Sigma_{\bar{V}_{k}}\right)\right)}
$$

In the formula, ${ }^{\Sigma_{\bar{V}_{k}}}$ represents the covariance matrix of the predicted residual $\bar{V}_{k}$, and $\operatorname{tr}(\cdot)$ is the trace operator of the matrix.

\section{2 selection of adaptive factors}

The adaptive factor models constructed according to the state model error discriminant statistics mainly include three-stage function adaptive factors, two-stage function adaptive factors and exponential function adaptive factors.

(1) three segment function adaptive factor [3]

$$
\alpha_{k}=\left\{\begin{array}{lr}
1 & \left|\Delta \overline{\boldsymbol{V}}_{k}\right| \leqslant c_{0} \\
\frac{c_{0}}{\left|\Delta \overline{\boldsymbol{V}}_{k}\right|}\left(\frac{c_{1}-\left|\Delta \overline{\boldsymbol{V}}_{k}\right|}{c_{1}-c_{0}}\right)^{2} & c_{0}<\left|\Delta \overline{\boldsymbol{V}}_{k}\right| \leqslant c_{1} \\
0 & \left|\Delta \overline{\boldsymbol{V}}_{k}\right|>c_{1}
\end{array}\right.
$$

Where $c_{0}$ and $c_{1}$ are constants, general $c_{0}=1.0 \sim 1.5, \quad c_{1}=3.0 \sim 8.5$

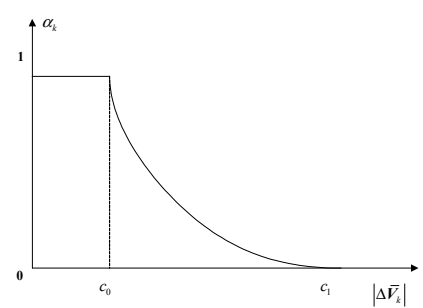

Fig. 1 adaptive function of three segment function

(2) two segment function adaptive factor $[4,5]$

$$
\alpha_{k}= \begin{cases}1 & \left|\Delta \bar{V}_{k}\right| \leqslant c \\ c /\left|\Delta \bar{V}_{k}\right| & \left|\Delta \bar{V}_{k}\right|>c\end{cases}
$$

Where $c$ is constants, $c=1.0 \sim 2.5$.

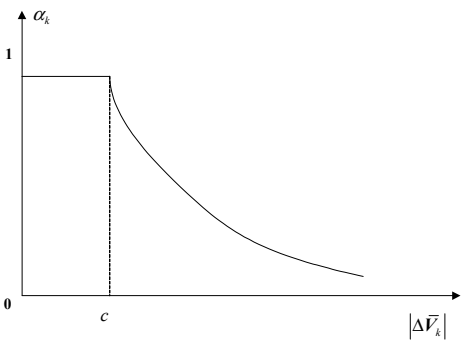

Fig. 2 adaptive function of two segment function (3) exponential function adaptive factor [6]

$$
\alpha_{k}= \begin{cases}1 & \left|\Delta \bar{V}_{k}\right| \leqslant c \\ e^{-\left(\left|\Delta \bar{V}_{k}-c\right|\right)^{2}} & \left|\Delta \bar{V}_{k}\right|>c\end{cases}
$$

Where $\mathrm{c}$ is constants, $\mathrm{c}=1.5$.

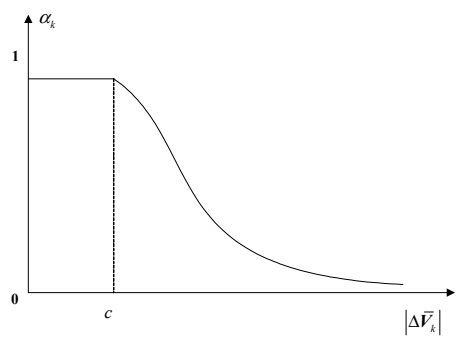

Fig. 3 exponential function adaptive factor

\section{3 robust estimation}

\section{1 definition and objectives of robust estimation}

Robust estimation (Robust Estimation) is also called robust estimation [7]. Unlike the least squares estimation, robust estimation is based on robust statistics and focuses on the actual robustness and reliability of the estimates, but does not require too much unbiasedness and validity of the estimates. In general, the stochastic control system runs and processes information. In the process, the received observation signals contain a variety of signals, in addition to the required useful signals, there are unwanted signals, such as random observation noise and interference signals. For this reason, when processing the actual observation data, it is necessary to deal with the observation noise and interference signal, and do robust estimation. Only in this way can the useful signal be effectively identified in the actual observation data, and the interference signal and noise signal be filtered out, and the optimal estimation of the parameters studied can be obtained [89].

Huber through quantitative analysis of Minimax robust solution [9], proposed three target of robust estimation[10] .

(1) Under the assumptions of the designed model, validity is the basic requirement for parameter estimation, in other words, the estimated value is in or near the optimal state.

(2) When there is a slight difference between the design model and the real model, the parameter estimation is less affected. 
(3) When there is a serious deviation between the design model and the real model, the parameter estimation will not be destructive.

Similarly, Hampel and others put forward the four target of robust estimation [11-12].

(1) the estimated value and observed samples should be in the best fitting state.

(2) when abnormal values appear, the estimation method should be identified better.

(3) When the object of study is an unbalanced design space, the estimation method can effectively identify the strong impact observation [13].

(4) when the structure related data and assumptions are in error, the estimation method can be processed.

From the definition and objective of robust estimation, it can be seen that when the design model or measurement value is inaccurate, robust estimation can ensure the reliability of the estimation at the cost of the validity and unbiased of the loss estimation. So the robust estimation is suitable for the state estimation problem when the system model is inaccurate.

\subsection{M estimation principle of robust estimation}

Discrete system is

$$
\begin{gathered}
\boldsymbol{x}_{k}=\boldsymbol{\Phi}_{k, k-1} \boldsymbol{x}_{k-1}+\boldsymbol{w}_{k-1} \\
\boldsymbol{z}_{k}=\boldsymbol{H}_{k} \boldsymbol{x}_{k}-\boldsymbol{v}_{k}
\end{gathered}
$$

Where $\boldsymbol{x}_{k}$ is the n-dimensional state vector of the ktime system, $\boldsymbol{z}_{k}$ is the m-dimensional measurement vector, $\boldsymbol{\Phi}_{k, k-1}$ is the one-step transition matrix from $t_{k-1}$ to $t_{k}, \boldsymbol{H}_{k}$ is the measurement matrix, $\boldsymbol{w}_{k}$ is the ndimensional process noise vector, $\boldsymbol{v}_{k}$ is the $\mathrm{m}$ dimensional measurement noise, and $\boldsymbol{w}_{k}$ and $\boldsymbol{v}_{k}$ are independent of each other.

In order to study the robust estimation properties of systems described in (9) and (10), the one-dimensional $\mathrm{M}$ estimation is introduced below.

Let $z_{1}, z_{2}, \cdots, z_{n}$ be the observation sample, $z_{i},(i=1,2, \cdots, n)$ be the observation value, and its distribution density function is $g\left(x-z_{i}\right)$. Maximum likelihood estimation requires state parameters to be satisfied: [14]

$$
\begin{gathered}
\Omega=\sum_{i=1}^{n} \ln g\left(x-z_{i}\right)=\max \\
\text { Instead of }-\ln g(\cdot)_{\text {with }} \rho(\cdot) \text {, we get: } \\
\Omega=\sum_{i=1}^{n} \rho\left(x-z_{i}\right)=\min
\end{gathered}
$$

the function $\rho(\cdot)$ is symmetric, continuous, strictly convex functions, or $\rho(\cdot)$ is a non descending function on the positive semi axis [15].

$\mathrm{M}$ is estimated as the solution to the above minimization problem. The derivation of formula (12) is equivalent to zero.

$$
\sum_{i=1}^{n} \phi\left(x-z_{i}\right)=0
$$

\section{3 the principle of robust estimation}

Consider the system described in formulas (9) and (10), and set up the measurement error equation.

$$
\boldsymbol{V}=\boldsymbol{H}_{k} \hat{\boldsymbol{x}}-\boldsymbol{z}
$$

Where $\boldsymbol{V}$ is a $m$ dimension residual vector and $\boldsymbol{H}_{k}$ is a $m \times n$ dimensional measurement matrix.

The extreme value function is obtained: $[126,132]$

$$
\sum_{i=1}^{n} \boldsymbol{p}_{i} \cdot \boldsymbol{\rho}(\boldsymbol{V})=\sum_{i=1}^{n} \boldsymbol{p}_{i} \cdot \boldsymbol{\rho}\left(\boldsymbol{h}_{i} \hat{\boldsymbol{x}}-\boldsymbol{z}_{i}\right)=\min
$$

Wher $\boldsymbol{h}_{i}$ is the $\boldsymbol{H}_{k}$ row vector of the $i_{\text {matrix, and }}$ $\boldsymbol{p}_{i}$ is the $\boldsymbol{P}$ line vector of the ${ }^{i}$ of the prior weight matrix. The derivative of $\boldsymbol{x}_{\text {is zero. }}$

$$
\sum_{i=1}^{n} \boldsymbol{p}_{i} \cdot\left[\frac{\partial}{\partial \boldsymbol{V}_{i}} \rho(\boldsymbol{V})\right] \cdot \frac{\partial \boldsymbol{V}_{i}}{\partial \boldsymbol{x}_{j}}=0
$$

In the formula, $\boldsymbol{V}_{i}$ is the residual of line $i$ of $\boldsymbol{V}$.

Make

$$
\frac{\partial}{\partial V_{i}} \rho(\boldsymbol{V})=\phi_{i}(\boldsymbol{V})
$$

derivation.

$$
\sum_{i=1}^{n} \boldsymbol{p}_{i} \cdot \phi_{i}(\boldsymbol{V}) \cdot \boldsymbol{h}_{i}=0
$$

Transform form (17) into matrix form [145].

$$
\boldsymbol{H}^{\mathrm{T}} \overline{\boldsymbol{P}} \boldsymbol{V}=0
$$

In the formula, $\overline{\boldsymbol{P}}$ is an equivalent weight matrix. Considering the formula (14), the robust estimation of parameter $\boldsymbol{x}_{\text {is obtained. }}$

$$
\hat{\boldsymbol{x}}=\left(\boldsymbol{H}_{k}^{\mathrm{T}} \overline{\boldsymbol{P}} \boldsymbol{H}_{k}\right)^{-1} \boldsymbol{H}_{k} \overline{\boldsymbol{P}} \boldsymbol{z}
$$

where

$$
\bar{p}_{i i}=\boldsymbol{p}_{i} \frac{\phi_{i}(\boldsymbol{V})}{\boldsymbol{V}_{i}}=\boldsymbol{p}_{i} \boldsymbol{\omega}_{i}
$$

\section{4 equivalent weight function}

(1) Tukey weight function [16] 


$$
\overline{\boldsymbol{P}}_{i}=\left(\begin{array}{ll}
\boldsymbol{p}_{i}\left[1-\left(\frac{\boldsymbol{V}_{i}}{c}\right)^{2}\right]^{2} & \left|\boldsymbol{V}_{i}\right| \leqslant c \\
0 & \left|\boldsymbol{V}_{i}\right|>c
\end{array}\right.
$$

Where the constant $\mathrm{C}$ is usually 4.685 . When the variance factor is unknown, it is 6 .

(2) Huber function [16]

$$
\overline{\boldsymbol{P}}_{i}=\left(\begin{array}{ll}
\boldsymbol{p}_{i} & \left|\boldsymbol{V}_{i}\right| \leqslant c \\
\boldsymbol{p}_{i} \frac{c}{\left|\boldsymbol{V}_{i}\right|} & \left|\boldsymbol{V}_{i}\right|>c
\end{array}\right.
$$

Where the constant $\mathrm{C}$ is usually 2 .

(3) Danish weight function [16]

$$
\overline{\boldsymbol{P}}_{i}=\left(\begin{array}{ll}
\boldsymbol{p}_{i} & \left|\boldsymbol{V}_{i}\right| \leqslant c \\
\boldsymbol{p}_{i} \exp \left[1-\left(\frac{\boldsymbol{V}_{i}}{c}\right)^{2}\right] & \left|\boldsymbol{V}_{i}\right|>c
\end{array}\right.
$$

Where the constant $\mathrm{C}$ is generally chosen to be 1.5 .

\section{4 precise intercept mathematical model of adjustable residual factor (AKUF)}

Through the measurement information provided by the inertial measurement element, the data related to the carrier can be calculated, such as the three-dimensional position, velocity and attitude information to the carrier. Therefore, with MIMU as the main system, MMW and IR as the auxiliary system, the position and attitude information of MIMU can be corrected to form the MIMU / MMW / IR composite guidance system.

(1) equation of state

In the guidance coordinate system, the state of the composite guidance system is selected.

$x=\left[\delta L, \delta, \delta h, \delta v_{\mathrm{E}}, \delta v_{\mathrm{N}}, \delta \dot{\mathrm{U}}_{\mathrm{U}}, \phi_{\mathrm{E}}, \phi_{\mathrm{N}}, \phi_{\mathrm{U}}, \varepsilon_{r x}, \varepsilon_{y y}, \varepsilon_{r z}, \nabla_{r x}, \nabla_{r y}, \nabla_{r z}\right]^{\mathrm{T}}$

$\delta L, \delta \lambda, \delta h$ is position error, $\delta v_{\mathrm{E}}, \delta v_{\mathrm{N}}, \delta v_{\mathrm{U}}$ is velocity error, $\phi_{\mathrm{E}}, \phi_{\mathrm{N}}, \phi_{\mathrm{U}}$ is attitude error, $\varepsilon_{r x}, \varepsilon_{r y}, \varepsilon_{r z}$ and $\nabla_{r x}, \nabla_{r y}, \nabla_{r z}$ are gyro error and accelerometer error respectively.

The equation of state is

$$
\dot{x}=f(x)+w
$$

where $f(x)$ is a nonlinear function of state quantity and $w$ is state error.

$$
f(x)=\left\{\begin{aligned}
& \boldsymbol{\alpha}=\boldsymbol{C}_{n b}^{-1}\left(I-\boldsymbol{C}_{n}^{c}\right) \hat{\boldsymbol{\omega}}_{i n}^{n}+\boldsymbol{C}_{n}^{c} \delta \hat{\boldsymbol{\omega}}_{i n}^{n}-\boldsymbol{C}_{b}^{c} \delta \hat{\boldsymbol{\omega}}_{i b}^{b} \\
& \delta \dot{v}_{3 \times 3}=\left[I-\left(\boldsymbol{C}_{n}^{c}\right)^{\mathrm{T}}\right] \boldsymbol{C}_{b}^{c} \hat{\boldsymbol{f}}_{s f}^{b}+\left(\boldsymbol{C}_{n}^{c}\right)^{\mathrm{T}} \boldsymbol{C}_{b}^{c} \delta \boldsymbol{f}_{s f}^{b}- \\
&\left(2 \delta \omega_{i e}^{n}+\delta \omega_{e n}^{n}\right) \times \boldsymbol{v}-\left(2 \hat{\omega}_{i e}^{n}+\hat{\omega}_{e n}^{n}\right) \times \delta \boldsymbol{v}+ \\
&\left(2 \omega_{i e}^{n}+\omega_{e n}^{n}\right) \times \delta \boldsymbol{v}+\delta \boldsymbol{g} \\
& \delta \dot{L}= \frac{\hat{v}_{N}^{n}}{\hat{R}_{M}+h}-\frac{\left(\hat{v}_{N}^{n}-\delta v_{N}^{n}\right)}{\left(\hat{R}_{M}-\delta R_{M}\right)+(\hat{h}-\delta h)} \\
& \delta \dot{\lambda}= \frac{\hat{v}_{E}^{n} \sec L}{\hat{R}_{N}+h}-\frac{\left(\hat{v}_{E}^{n}-\delta v_{E}^{n}\right) \sec (\hat{L}-\delta L)}{\left(\hat{R}_{N}-\delta R_{N}\right)+(\hat{h}-\delta h)} \\
& \delta \dot{h}= \delta v_{U}^{n}
\end{aligned}\right.
$$

where $C_{n}^{c}$ is the attitude transformation matrix from navigation coordinate system (n) to computational coordinate system (c), $\boldsymbol{C}_{b}^{c}$ body coordinate system (b) to C system; $\hat{\omega}_{i e}^{n}$ is the projection of the actual value of angular velocity from earth coordinate system (e) to geocentric inertial coordinate system (i) on n system; $\omega_{i e}^{n}$ is the projection of the ideal value of angular velocity from e to I system. $\delta \omega_{i n}^{n}$ and $\delta \omega_{i b}^{b}$ is the calculation error of $\omega_{i n}^{n}$ and $\omega_{i b}^{b}$ respectively; the specific force output of $\hat{\boldsymbol{f}}_{s f}^{b}$ and $\boldsymbol{\delta} \boldsymbol{f}_{s f}^{b}$ actual accelerometer and the measurement error of accelerometer; the parameters in the above formula are listed in literature [17].

(2) measurement equation

The position and attitude information obtained by MMW measurement is

$$
\left[\begin{array}{c}
r_{\mathrm{MMW}} \\
\psi_{\mathrm{MMW}} \\
\theta_{\mathrm{MMW}}
\end{array}\right]=\left[\begin{array}{c}
\sqrt{x^{2}+y^{2}+z^{2}} \\
\arctan (y / x) \\
\arctan \left(z / \sqrt{x^{2}+y^{2}}\right)
\end{array}\right]
$$

Where $r, \psi$ and $\theta$ are information about distance, azimuth and pitch angle of missile to target respectively. The three-dimensional position and some attitude information of the missile relative to the target can be obtained by the equations, and then the MIMU / MMW measurement model can be obtained.

$$
Y_{1}=\left[\begin{array}{c}
L_{\mathrm{IMU}}-L_{\mathrm{MMW}} \\
\lambda_{\mathrm{IMU}}-\lambda_{\mathrm{MMW}} \\
h_{\mathrm{IMU}}-h_{\mathrm{MMW}} \\
\psi_{\mathrm{IMU}}-\psi_{\mathrm{MMW}} \\
\theta_{\mathrm{IMU}}-\theta_{\mathrm{MMW}}
\end{array}\right]=\left[\begin{array}{c}
\delta L_{1} \\
\delta \lambda_{1} \\
\delta h_{1} \\
\delta \psi_{1} \\
\delta \theta_{1}
\end{array}\right]+\left[\begin{array}{c}
v_{1 L} \\
v_{1 \lambda} \\
v_{1 h} \\
v_{1 \psi} \\
v_{1 \theta}
\end{array}\right]
$$

The subscript IMU represents the information obtained by MIMU measurement, the subscript MMW is the information obtained by millimeter wave 
measurement, and the $v_{1 L}, v_{1 \lambda}, v_{1 h}, v_{1 \psi}$ and $v_{1 \theta}$ are the measurement errors of position, azimuth and pitch of the MIMU/MMW subsystem.

Similarly, the attitude information can be obtained through

$$
\begin{gathered}
{\left[\begin{array}{c}
\psi_{\mathrm{IR}} \\
\theta_{\mathrm{IR}}
\end{array}\right]=\left[\begin{array}{c}
\arctan (y / x) \\
\arctan \left(z / \sqrt{x^{2}+y^{2}}\right)
\end{array}\right]} \\
Y_{2}=\left[\begin{array}{c}
\psi_{\mathrm{IMU}}-\psi_{\mathrm{IR}} \\
\theta_{\mathrm{IMU}}-\theta_{\mathrm{IR}}
\end{array}\right]=\left[\begin{array}{c}
\delta \psi_{2} \\
\delta \theta_{2}
\end{array}\right]+\left[\begin{array}{c}
v_{2 \psi} \\
v_{2 \theta}
\end{array}\right]
\end{gathered}
$$

\section{5 filtering data optimization}

Data registration is an unavoidable and important problem in multi-sensor information fusion. The difficulty in this problem is how to unify the time and space of each sensor into the reference standard time and space, namely time synchronization and space registration.

\section{1 non equal interval time update problem}

Time synchronization is the premise of multi-sensor information fusion. Maintaining high-precision time synchronization among sensors is the basis of improving data processing accuracy. In MIMU/MMW/IR hybrid guidance system, the data update rate of inertial devices is much higher than that of millimeter wave and infrared subsystems. Therefore, considering the non-coincidence between the measurement output time of the main system and the measurement output time of the subsystem, the minimum common times of the data update of each sensor are obtained under the assumption that the filtering period is within the time period. The number is the sampling period of filtering, as shown in Figure 4.

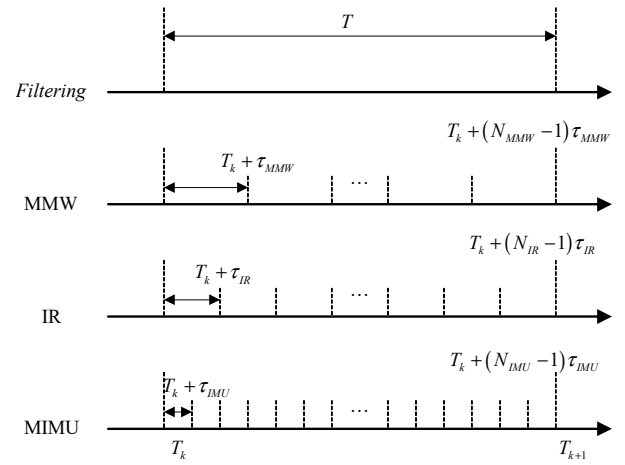

Fig. 4 synchronization diagram of filter period and sensor sampling period

\section{2 space registration problem}

Due to the different location of sensors, the sensors work in different measuring coordinate systems. Before data fusion, data from different sensors need to be processed. The processing includes coordinate transformation and error calibration. Coordinate system change is to map the position information of the known sensor in the measurement coordinate system to the reference coordinate system. Error calibration usually estimates the offset of each sensor by testing the target with known location information and then corrects it. The principle is as follows

$$
\left\{\begin{array}{l}
\delta_{r, k}=r_{k}-r=\Delta r_{k}+\varepsilon_{r} \\
\delta_{\psi, k}=\psi_{k}-\psi=\Delta \psi_{k}+\varepsilon_{\psi} \\
\delta_{\theta, k}=\theta_{k}-\theta=\Delta \theta_{k}+\varepsilon_{\theta}
\end{array}\right.
$$

In the rectangular coordinate system, the moving mode of the target in space is maneuvering. When the time is zero, the relative position is $[50 \mathrm{~km}, 30 \mathrm{~km}, 15 \mathrm{~km}]$, the relative velocity is $[-1800 \mathrm{~m} / \mathrm{s}, 1200 \mathrm{~m} / \mathrm{s}, 500 \mathrm{~m} / \mathrm{s}]$, the acceleration vector is $[5 \mathrm{~g}, 2 \mathrm{~g}, \mathrm{~g}]$, and the track of the missile relative to the target is shown in Figure 6 . The sampling times of MIMU, MMW and IR sensors are $0.01 \mathrm{~s}, \quad 0.1 \mathrm{~s}$ and $0.2 \mathrm{~s}$, respectively; gyro drift is $\left.0.1^{\circ}\right) / \sqrt{h}$, zero offset is $\left.0.01^{(}\right) / \sqrt{h}$; constant error is $10-3 \mathrm{~g}$; horizontal error root mean square is $5 \mathrm{~m}$, height error root mean square is $8 \mathrm{~m}$; MMW ranging error standard deviation is $20 \mathrm{~m}$, attitude error is $0.01 \mathrm{rad}$; and IR angle error is $0.002 \mathrm{rad}$. The period is $0.2 \mathrm{~S}$ and the observation time is $20 \mathrm{~s}$.

$$
\left\{\begin{array}{l}
\Delta r_{k}=\frac{1}{n} \sum_{i=1}^{n} \delta_{r, k}(i) \\
\Delta \psi_{k}=\frac{1}{n} \sum_{i=1}^{n} \delta_{\psi, k}(i) \\
\Delta \theta_{k}=\frac{1}{n} \sum_{i=1}^{n} \delta_{\theta, k}(i)
\end{array}\right.
$$

\section{6 simulation experiment and analysis}

The formula (33) nonlinear target three-dimensional relative motion model is used as the system state model [15].

$$
\left\{\begin{array}{l}
\dot{x}_{1}=x_{2} \\
\dot{x}_{2}=\boldsymbol{r} x_{6}^{2} \cos ^{2} x_{3}+x_{1} x_{4}^{2}+\boldsymbol{a}_{T i}-\boldsymbol{a}_{M i} \\
\dot{x}_{3}=x_{4} \\
\dot{x}_{4}=-2 \frac{x_{2} x_{4}}{x_{1}}+\frac{\boldsymbol{a}_{T k}-\boldsymbol{a}_{M k}}{x_{1}}-\frac{x_{6}^{2} \sin ^{2} 2 x_{3}}{2} \\
\dot{x}_{5}=x_{6} \\
\dot{x}_{6}=-2 \frac{x_{2} x_{6}}{x_{1}}+\frac{\boldsymbol{a}_{T j}-\boldsymbol{a}_{M j}}{x_{1}}+2 x_{4} x_{6} \tan x_{3}
\end{array}\right.
$$

Where $x_{1}=r, \quad x_{2}=\dot{r}, x_{3}=\theta_{L}, x_{4}=\dot{\theta}_{L}$, $x_{5}=\psi_{L}, \quad x_{6}=\dot{\psi}_{L}$, as shown in Figure 5 . 


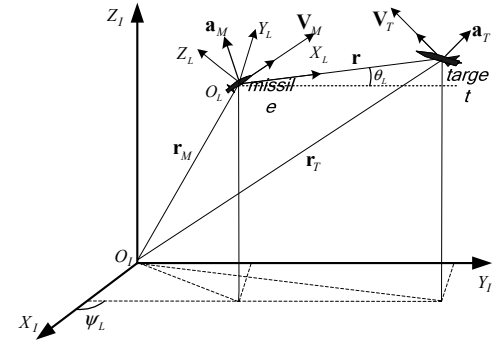

Fig. 5 relative kinematic relations of projectiles and 3D objects

In a rectangular coordinate system, the target is maneuvering in space. The relative position of the initial time is $[50 \mathrm{~km}, 30 \mathrm{~km}, 15 \mathrm{~km}]$, the relative velocity is [$1800 \mathrm{~m} / \mathrm{s}, 1200 \mathrm{~m} / \mathrm{s}, 500 \mathrm{~m} / \mathrm{s}]$, and the acceleration vector is $[5 \mathrm{~g}, 2 \mathrm{~g}, \mathrm{~g}]$. The track of the missile relative to the target is shown in Figure 6.6. The sampling times of MIMU, MMW and IR sensors are $0.01 \mathrm{~s}, 0.1 \mathrm{~s}$ and $0.2 \mathrm{~s}$, respectively; gyro drift is $\left.0.1^{(}\right) / \sqrt{h}$, zero offset is $\left.0.01^{\circ}\right) / \sqrt{h}$; constant error is $10-3 \mathrm{~g}$; horizontal error root mean square is $5 \mathrm{~m}$, height error root mean square is $8 \mathrm{~m}$; MMW ranging error standard deviation is $20 \mathrm{~m}$, attitude error is $0.01 \mathrm{rad}$; and IR angle error is $0.002 \mathrm{rad}$. The period is $0.2 \mathrm{~S}$ and the observation time is $20 \mathrm{~s}$.

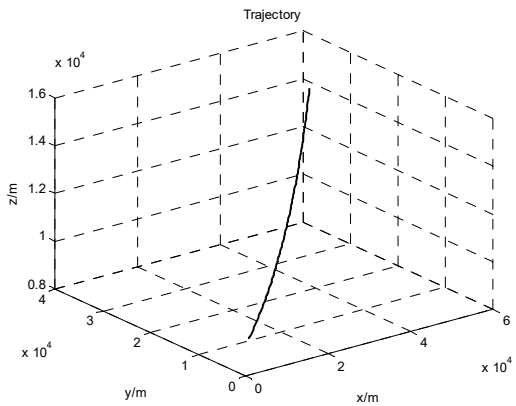

Fig. 6 track chart of relative motion between missile and target

Adaptive UKF, standard EKF and UKF are applied to the MIMU / MMW / IR compound guidance system for simulation verification. The simulation results of location information are shown in Figure 7 to Figure 9. The simulation results show that, firstly, the position error of standard EKF is the largest, the standard UKF is significantly reduced, and the position error of AUKF is the smallest. This is because this paper adopts a system model with high degree of nonlinearity, because in the calculation, the Taylor expansion of EKF can only be taken as a first order approximation, there is a large rounding error. When the system error is Gaussian error, the UKF algorithm is at least second-order approximation, so the simulation conditions are the same. Compared with the standard EKF algorithm, the AUKF algorithm and the standard UKF algorithm have significant advantages in filtering accuracy.

Secondly, the horizontal position error is less than the height position error, that is, the filtering results of the three algorithms in Figures 7 and 8 are better than those in Figure 9. This is due to the instability of inertial navigation system in height direction. However, the filtering results do not diverge because the filtering measurements also incorporate the information provided by MMW and IR.

Finally, from Figure 7 to Figure 9, it can be seen that the filtering results fluctuate greatly in the initial stage of simulation using standard EKF and standard UKF algorithm, while the AUKF algorithm can make the filtering results output smoothly earlier and improve the convergence speed of the UKF algorithm. It shows that the adjustable residual factor can be used to discriminate statistics by compensating state model errors. It can effectively improve the anti disturbance ability of the system.

Finally, from Figure 7 to Figure 9, it can be seen that the filtering results fluctuate greatly in the initial stage of simulation using standard EKF and standard UKF algorithm, while the AUKF algorithm can make the filtering results output smoothly earlier and improve the convergence speed of the UKF algorithm. It shows that the adjustable residual factor can be used to discriminate statistics by compensating state model errors. It can effectively improve the anti disturbance ability of the system.

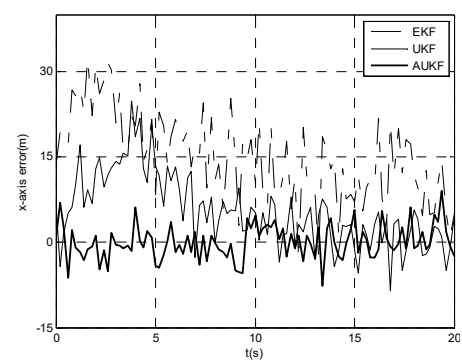

Fig. $7 \mathrm{X}$ axis position error curve

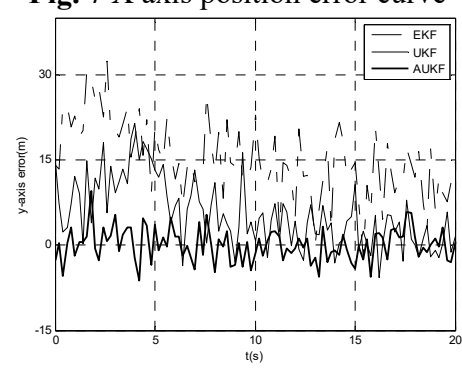

Fig. $8 \mathrm{Y}$ axis position error curve

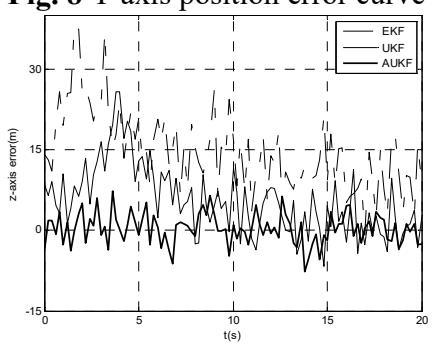

Fig. $9 \mathrm{Z}$ axis position error curve

Compared with EKF, the position error obtained by standard EKF is the largest, and the mean square error is less than $24 \mathrm{M}$. The position error obtained by standard UKF is slightly smaller than that obtained by EKF, and the position mean square error is less than $13 \mathrm{M}$. The adaptive UKF algorithm proposed in this paper has relatively small position error and fast convergence speed, and the position mean square error is stable within $6 \mathrm{~m}$. The specific values are shown in Table 6.1. Therefore, the relative position error estimation accuracy 
of the adaptive UKF algorithm proposed in this paper is higher than that of the standard EKF and UKF algorithms, which can improve the missile strike accuracy.

Table 1 mean absolute error of three algorithms

\begin{tabular}{|c|c|c|c|}
\hline $\begin{array}{l}\text { Wave } \\
\text { filtering } \\
\text { Method }\end{array}$ & $\begin{array}{l}\mathrm{X} \text { axis } \\
\text { position } \\
\text { error }\end{array}$ & $\begin{array}{l}\mathrm{Y} \text { axis } \\
\text { position } \\
\text { error }\end{array}$ & $\begin{array}{c}\mathrm{Z} \text { axis } \\
\text { position } \\
\text { error }\end{array}$ \\
\hline EKF & $22.515 \mathrm{~m}$ & $22.143 \mathrm{~m}$ & $23.659 \mathrm{~m}$ \\
\hline UKF & $10.762 \mathrm{~m}$ & $10.823 \mathrm{~m}$ & $12.372 \mathrm{~m}$ \\
\hline AUKF & $4.834 \mathrm{~m}$ & $4.903 \mathrm{~m}$ & $6.227 \mathrm{~m}$ \\
\hline
\end{tabular}

\section{7 peroration}

Firstly, the principle of adaptive filtering and robust estimation is analyzed. On this basis, an adaptive Unscented Kalman filter algorithm with adjustable residual factor is designed. This algorithm uses predictive residual to construct state model error statistics, and adjusts the contribution of state model information to state parameter estimation by adaptive factor. The influence of noise state on the estimation of state parameters is controlled. The time synchronization and spatial registration of heterogeneous sensors are studied, and the filtering data is optimized. The proposed algorithm is applied to MIMU/MMW/IR compound guidance system for simulation and verification. The results show that the proposed multi-source asynchronous information fusion algorithm is more accurate than the standard EKF and UKF algorithms in calculating the position errors between missiles and targets in the midcourse of guidance, and can effectively improve the precision of missile guidance.

\section{References}

1. Gao Yi, Gao She Sheng. Robust adaptive Sage filtering and its application in integrated navigation [J].TT\&amp;C technology, 2015,4:135-141.

2. Xing Yexin, Lou Guowei, Li Xingguo, et al. Research on passive detection of stealth targets in the air by millimeter wave [J].Journal of Nanjing University of Technology, 2011, 35 (3): 289-293.

3. Bowyer, R S Bogner. Cooperative behavior in Multi-sensor systems [EB/OL]. http://www.go.com/, 1999.

4. [4] Zhang Weiming. Intelligent Collaborative Information Technology [M]. Beijing: Publishing House of electronics industry, 2002.

5. B.Horling, V.Lesser. A survey of mlulti-agent organizational paradigms $[\mathrm{J}]$. The Kowledge Engineering Review, 2005, 19(4): 281-316.
6. Yang Yu PU. Overview of multi-agent collaboration technology $[\mathrm{J}]$. information and control, 2001, 23 (4): 337-342.

7. Yan Dong-mei, Wang Jin-kuan, Gu De-ying. Target tracking using WSN based on multiagent coordination method[C]. The 7th World Congress on Intelligent Control and Automation, Chongqing, China, June 25-27, 2008.

8. Chen Jianxia, Yu Haibin. A Dynamic Alliance Updating Mechanism for Cooperative Task Assignment in Wireless Sensor Networks [J].Journal of Sensing Technology, 2009, 22 (4): 501-502.

9. Lu Lei, Xu Ming, Lin Yongduo, Xia Desheng. Modeling and algorithm comparison of state estimation for microgrid power system [J]. Electrical applications. 2013.32 (04): 66-69.

10. Qiu Fengyun Kalman filtering theory and its application in communication and signal processing [D].Jinan: Master thesis of Shandong University, 2008.

11. J.M. Molina Lopez, J R Casar Corredera. Cooperative management of netted surveillance sensors [C]. IEEE International Conference on system, Man and Cybernetics, Oct. 12-15, 1997, Vol. 1:845-850.

12. Fan Bo, Pan Quan, Zhang Hongcai, et al. Introducing multi-agent technology into information fusion $[\mathrm{J}]$. Computer engineering and application, 2003, 22:100-102.

13. Zhang Guangyuan, Wang Fujun, Wei Zhensheng. Agent's Multi-Sensor Management Scheme [J]. Firepower and Command Control, 2009, 34 (7): 168-170.

14. Chen Mimi, Jiang Gangwu, Jiang Ting. Optical Survey of Space Cooperation Target Rendezvous and Docking [J].Surveying and Mapping Science, 2008, 10 (33): 30-32.

15. Cheng Hongbin, Zhang Xiaofeng, Zhang Fengming. Research on Agent-based multi-sensor management prototype system [J]. Modern defense technology, 2006, 34 (2): 44-50.

16. Liu Dan.Research on robust estimation method for signal reconstruction of nonlinear multifunctional sensors[J].Journal of Sensing Technology, 2008,21(10): 1721-1725.

17. Li Wei, Zhang Fengming.Research and Design of Sensor Management System Based on MultiAgent[J].Sensors and Instruments, 2006, 22 (7-1): 157-159. 